\title{
Clinical Mortality in a Large COVID-19 Cohort: Observational Study
}

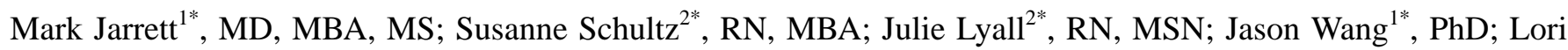
Stier $^{2^{*}}, \mathrm{RN}, \mathrm{EdD}$; Marcella De Geronimo ${ }^{3 *}$, MS; Karen Nelson ${ }^{2 *}, \mathrm{RN}, \mathrm{MBA}$

${ }^{1}$ Donald and Barbara Zucker School of Medicine at Hofstra/Northwell, Hofstra University, Hempstead, NY, United States

${ }^{2}$ Institute for Clinical Excellence and Quality/Safety, Northwell Health, New Hyde Park, NY, United States

${ }^{3}$ Krasnoff Quality Management Institute, Northwell Health, New Hyde Park, NY, United States

*all authors contributed equally

Corresponding Author:

Mark Jarrett, MD, MBA, MS

Donald and Barbara Zucker School of Medicine at Hofstra/Northwell

Hofstra University

500 Hofstra University

Hempstead, NY, 11549

United States

Phone: 15163216044

Email: MJarrett@northwell.edu

\section{Abstract}

Background: Northwell Health, an integrated health system in New York, has treated more than 15,000 inpatients with COVID-19 at the US epicenter of the SARS-CoV-2 pandemic.

Objective: We describe the demographic characteristics of patients who died of COVID-19, observation of frequent rapid response team/cardiac arrest (RRT/CA) calls for non-intensive care unit (ICU) patients, and factors that contributed to RRT/CA calls.

Methods: A team of registered nurses reviewed the medical records of inpatients who tested positive for SARS-CoV-2 via polymerase chain reaction before or on admission and who died between March 13 (first Northwell Health inpatient expiration) and April 30, 2020, at 15 Northwell Health hospitals. The findings for these patients were abstracted into a database and statistically analyzed.

Results: Of 2634 patients who died of COVID-19, 1478 (56.1\%) had oxygen saturation levels $\geq 90 \%$ on presentation and required no respiratory support. At least one RRT/CA was called on 1112/2634 patients (42.2\%) at a non-ICU level of care. Before the RRT/CA call, the most recent oxygen saturation levels for 852/1112 (76.6\%) of these non-ICU patients were at least $90 \%$. At the time the RRT/CA was called, 479/1112 patients (43.1\%) had an oxygen saturation of $<80 \%$.

Conclusions: This study represents one of the largest reviewed cohorts of mortality that also captures data in nonstructured fields. Approximately 50\% of deaths occurred at a non-ICU level of care despite admission to the appropriate care setting with normal staffing. The data imply a sudden, unexpected deterioration in respiratory status requiring RRT/CA in a large number of non-ICU patients. Patients admitted at a non-ICU level of care suffered rapid clinical deterioration, often with a sudden decrease in oxygen saturation. These patients could benefit from additional monitoring (eg, continuous central oxygenation saturation), although this approach warrants further study.

(J Med Internet Res 2020;22(9):e23565) doi: 10.2196/23565

\section{KEYWORDS}

COVID-19; mortality; respiratory failure; hypoxemia; observational; review; cohort; ICU; intensive care unit 


\section{Introduction}

Downstate New York was the first epicenter of the SARS-CoV-2 pandemic in the United States [1,2]. Northwell Health, an integrated health system, has treated more than 15,000 inpatients with COVID-19. Comprehensively analyzing the characteristics of patients who die of COVID-19 can help define the clinical nature of COVID-19 infection and potentially suggest new care protocols. For 7 years, Northwell Health has used a centralized mortality review process with data validated through rigorous internal review and high interrater reliability (92\% to $96 \%$ ). This robust process was applied to a customized database to review all 2634 patients who died of COVID-19 in Northwell Health's adult acute care hospitals between March and April 2020. During this overwhelming surge, documentation was made in various notes as well as in structured fields in the electronic health record (EHR) systems. This study describes the demographic characteristics of patients who died of COVID-19 and the observation of frequent rapid response team/cardiac arrest (RRT/CA) calls for patients not in the intensive care unit (ICU). We also discuss factors that contributed to the RRT/CA calls, which may be a significant element in planning for a resurgence of the pandemic.

\section{Methods}

\section{Study Design}

Northwell Health is New York State's largest health care provider and private employer. With 23 hospitals (including specialty hospitals) and nearly 800 outpatient practice sites, the organization cares for over 2 million people in greater metropolitan New York. A team of registered nurses in the corporate quality department retrospectively reviewed medical records from 15 acute care hospitals. This team routinely conducts clinical reviews of all adult acute inpatient mortalities (approximately 5000 per year). A physician advisor was available to the team to consult on clinical questions.

Database elements were based on Northwell Health's experience with treating patients with COVID-19, literature review from countries that had early experience in treating patients, and clinical trials being conducted at the Feinstein Institutes for Medical Research. Also, the data were captured in the database established under the direction of critical care intensivists at the epicenter of the pandemic, other subject matter experts, and quality leadership. During data abstraction, modifications and enhancements were made to the database based on trends and emerging information. The demographic data, comorbidities, clinical findings, and management of COVID-19 patients who died were analyzed.

\section{Patient Characteristics}

The analyzed cases included inpatients who tested positive for SARS-CoV-2 via polymerase chain reaction before or on admission and who then died between March 13 (first Northwell
Health inpatient death) and April 30, 2020. Emergency department (ED) mortalities were excluded. Demographic data and comorbidities were abstracted from the medical records of admitted patients. Initially, data were collected on 10 patient comorbidities that were deemed important and were then narrowed down to 6 comorbidities for inclusion based on our initial analysis. Transfers from one in-system hospital to another were merged and considered as a single visit. Notable patient outcomes that were measured were the level of ICU care (validated and abstracted from the provider order) and a call for RRT/CA. The Institutional Review Board of Northwell Health deemed this study as exempt and waived the requirement for informed consent.

\section{Statistical Analysis}

Statistical analyses were performed using chi-square tests for categorical variables and $t$ tests for continuous variables. A multivariable logistic regression model was created to determine independent risk factors for the outcome variables. Statistical significance was considered at $P<.05$. All statistical analyses were performed in SAS v9.4 (SAS Institute).

\section{Data Sharing}

The data that support the findings of this study are available on request from COVID19@northwell.edu. The data are not publicly available due to restrictions, as this could compromise the privacy of the research participants.

\section{Results}

\section{Patient Characteristics}

The baseline characteristics of the 2634 patients who died of COVID-19 are described in Tables 1-3. The age range was 21-107 years in the following categories: 21 to 39 years $(49 / 2634,1.9 \%), 40$ to 59 years $(351 / 2634,13.3 \%), 60$ to 79 years $(1241 / 2634,47.1 \%)$, and $\geq 80$ years $(993 / 2634,37.7 \%)$. In the patient cohort, $1664 / 2634$ patients $(63.2 \%)$ were male and $970 / 2634(36.8 \%)$ were female. Among the 2634 patients, $1256(47.7 \%)$ were White, $463(17.6 \%)$ were Black, $230(8.7 \%)$ were Asian, and $685(26.0 \%)$ were of other/unknown race. The majority of patients $(1839 / 2634,69.8 \%)$ reported Medicare as their insurance. The most common comorbidities among these patients were hypertension $(1719 / 2634,65.3 \%)$, diabetes (1043/2634, 39.6\%), and dementia (431/2634, 16.4\%). Fewer patients had chronic obstructive pulmonary disease (385/2634, $14.6 \%)$, heart failure $(291 / 2634,11.1 \%)$, and end stage renal disease $(166 / 2634,6.3 \%)$. Of these six comorbidities, more than half of the patients $(1350 / 2634,51.3 \%)$ had 2 or more comorbidities, and 445/2634 (16.9\%) had 0 comorbidities. The majority of patients with a known BMI, calculated as weight in kilograms divided by height in meters squared, of 25 or more were categorized as follows: 25 to $29.99(732 / 2634,27.8 \%)$, 30 to $34.99(401 / 2634,15.2 \%), 35$ to $39.99(190 / 2634,7.2 \%)$, and $\geq 40(147 / 2634,5.6 \%)$. 
Table 1. Baseline characteristics of patients hospitalized with COVID-19 who died (N=2634), $\mathrm{n}(\%)$.

\begin{tabular}{|c|c|}
\hline Baseline characteristic & Value \\
\hline \multicolumn{2}{|l|}{ Age (years) } \\
\hline $21-39$ & $49(1.86)$ \\
\hline $40-59$ & $351(13.3)$ \\
\hline $60-79$ & $1241(47.1)$ \\
\hline$\geq 80$ & $993(37.7)$ \\
\hline \multicolumn{2}{|l|}{ Sex } \\
\hline Male & $1664(63.2)$ \\
\hline Female & $970(36.8)$ \\
\hline \multicolumn{2}{|l|}{ Race } \\
\hline White & $1256(47.7)$ \\
\hline Black & $463(17.6)$ \\
\hline Asian & $230(8.7)$ \\
\hline Other/unknown & $685(26.0)$ \\
\hline \multicolumn{2}{|l|}{ Payment method } \\
\hline Commercial insurance & $413(15.7)$ \\
\hline Medicaid & $341(13.0)$ \\
\hline Medicare & $1839(69.8)$ \\
\hline Self-pay & $41(1.6)$ \\
\hline \multicolumn{2}{|l|}{ Comorbidities } \\
\hline Hypertension & $1719(65.3)$ \\
\hline $\mathrm{COPD}^{\mathrm{a}}$ & $385(14.6)$ \\
\hline Diabetes & $1043(39.6)$ \\
\hline Heart failure & $291(11.1)$ \\
\hline Dementia & $431(16.4)$ \\
\hline End stage renal disease & $166(6.3)$ \\
\hline \multicolumn{2}{|l|}{ Number of comorbidities } \\
\hline 0 & $445(16.9)$ \\
\hline 1 & 839 (31.9) \\
\hline 2 & $934(35.5)$ \\
\hline 3 & $343(13.0)$ \\
\hline 4 & $66(2.5)$ \\
\hline 5 & $7(0.3)$ \\
\hline 6 & $0(0.0)$ \\
\hline \multicolumn{2}{|l|}{ BMI $\left(\mathrm{kg} / \mathrm{m}^{2}\right)$} \\
\hline Unknown & $494(18.8)$ \\
\hline$<18.5$ & $82(3.1)$ \\
\hline $18.5-24.99$ & $588(22.3)$ \\
\hline $25-29.99$ & $732(27.8)$ \\
\hline $30-34.99$ & $401(15.2)$ \\
\hline $35-39.99$ & $190(7.2)$ \\
\hline$\geq 40$ & $147(5.6)$ \\
\hline
\end{tabular}


${ }^{\mathrm{a} C O P D}$ : chronic obstructive pulmonary disease. 
Table 2. Hospitalization characteristics of patients hospitalized with COVID-19 who died (N=2634), n (\%).

\begin{tabular}{|c|c|}
\hline Hospitalization characteristic & Value \\
\hline \multicolumn{2}{|l|}{ Admission source } \\
\hline Home & $1895(72.0)$ \\
\hline Rehabilitation & $127(4.8)$ \\
\hline Skilled nursing facility & $411(15.6)$ \\
\hline Transfer from another acute care hospital & $201(7.6)$ \\
\hline \multicolumn{2}{|l|}{ Emergency department visit } \\
\hline Within 48 hours of this admission & $51(1.9)$ \\
\hline Within 7 days of this admission & $125(4.8)$ \\
\hline \multicolumn{2}{|l|}{ Readmission } \\
\hline Within 24 hours & $20(0.8)$ \\
\hline Within 7 days & $75(2.9)$ \\
\hline Within 30 days & $194(7.4)$ \\
\hline \multicolumn{2}{|l|}{ Level of care at time of death } \\
\hline $\mathrm{ICU}^{\mathrm{a}}$ & $1299(49.3)$ \\
\hline Non-ICU & $1335(50.7)$ \\
\hline \multicolumn{2}{|l|}{ Level of care at time of admission } \\
\hline ICU & $541(20.5)$ \\
\hline Medical/surgical unit & $1230(46.7)$ \\
\hline Telemetry/stepdown unit & $863(32.8)$ \\
\hline \multicolumn{2}{|l|}{ Overall length of stay (days) } \\
\hline $0-7$ & $1420(53.9)$ \\
\hline$\geq 8$ & $1214(46.1)$ \\
\hline \multicolumn{2}{|l|}{ ICU length of stay (days) } \\
\hline $0-7$ & $872(33.1)$ \\
\hline$\geq 8$ & $574(21.8)$ \\
\hline \multicolumn{2}{|l|}{ Oxygen saturation on presentation (\%) } \\
\hline$<80$ & $459(17.4)$ \\
\hline $80-89.9$ & $667(25.3)$ \\
\hline$\geq 90$ & $1478(56.1)$ \\
\hline Unable to determine & $30(1.2)$ \\
\hline \multicolumn{2}{|l|}{ Initial respiratory support on presentation } \\
\hline None & $1397(53.0)$ \\
\hline Nasal cannula & $363(13.8)$ \\
\hline Nonrebreather mask & $742(28.2)$ \\
\hline Ventilator & $24(0.9)$ \\
\hline High-flow nasal cannula & $8(0.3)$ \\
\hline Ventimask & $11(0.4)$ \\
\hline BiPAP $^{b}$ & $13(0.5)$ \\
\hline Other & $27(1.0)$ \\
\hline Unable to determine & $49(1.9)$ \\
\hline $\mathrm{RRT} / \mathrm{CA}^{\mathrm{d}}$ while not at ICU level of care & $1112(42.2)$ \\
\hline
\end{tabular}




\begin{tabular}{ll}
\hline Hospitalization characteristic & Value \\
\hline Proning & $756(28.7)$ \\
Yes & $1878(71.3)$ \\
No & $191(25.3)$ \\
$\quad$ Proning without mechanical ventilation $(\mathrm{n}=756)$ & $213(28.2)$ \\
$\quad$ Proning prior to mechanical ventilation $(\mathrm{n}=756)$ & $214(28.3)$ \\
$\quad$ Proning during mechanical ventilation $(\mathrm{n}=756)$ & $138(18.3)$ \\
$\quad$ Proning prior to and during mechanical ventilation(n=756) & $1631(61.9)$ \\
DNR ${ }^{\mathrm{d}}$ complete & $1014(38.5)$ \\
Palliative care consult & $114(4.3)$ \\
Clinical trial inclusion & \\
\hline
\end{tabular}

${ }^{\mathrm{a}}$ ICU: intensive care unit.

${ }^{\mathrm{b}} \mathrm{BiPAP}$ : bilevel positive airway pressure

${ }^{\mathrm{c}} \mathrm{RRT} / \mathrm{CA}$ : rapid response team/cardiac arrest.

${ }^{\mathrm{d}}$ DNR: do not resuscitate.

Table 3. Mechanical ventilation characteristics of patients hospitalized with COVID-19 who died.

\begin{tabular}{llll}
\hline Mechanical ventilation characteristic & $\mathrm{n}$ & $\begin{array}{l}\% \\
\text { Total patients (N=2634) }\end{array}$ & $\begin{array}{l}\text { Patients who were ventilated } \\
(\mathrm{n}=1403)\end{array}$ \\
\hline Traditional ventilator & & 47.9 & 89.7 \\
Converted BiPAP & & 0.1 & 10.1 \\
Anesthesia machine & 14259 & 0.08 & 0.1 \\
Increased oxygen requirement prior to mechanical ventilation & 1332 & 50.6 & 94.9 \\
Mechanical ventilation length, days & 2 & & 60.7 \\
$\quad 0-7$ & 851 & 32.3 & 39.3 \\
$\quad 28$ & 552 & 20.9 & 19.2 \\
\hline
\end{tabular}

abiPAP: bilevel positive airway pressure.

\section{Patient Outcomes}

Most patients were admitted from home (1895/2634, 71.9\%). The remaining patients were admitted from a skilled nursing facility $(411 / 2634,15.6 \%)$, an acute care facility (201/2634, $7.6 \%)$, or a rehabilitation facility $(127 / 2634,4.8 \%)$. The percentage of patients with a prior ED visit within 7 days of admission was $4.8 \%(125 / 2634)$, and that of patients with a prior ED visit within 48 hours of admission was 1.9\% (51/2634). The percentage of patients readmitted within 30 days was $7.4 \%$ (194/2634), 2.9\% (75/2634) were readmitted within 7 days, and $0.8 \%(20 / 2634)$ were readmitted within 24 hours. On presentation, most patients $(1478 / 2634,56.1 \%)$ had an oxygen saturation level greater than or equal to $90 \%$, and more than half $(1397 / 2634,53.0 \%)$ required no respiratory support. Others required a nasal cannula $(363 / 2634,13.8 \%)$, a nonrebreather mask $(742 / 2634,28.2 \%)$, or mechanical ventilation $(24 / 2634$, $0.9 \%)$. More than half of the patients who died (1403/2634, $53.2 \%$ ) required mechanical ventilation during their clinical course. Of those 1403 patients, 1332 (94.9\%) had increasing oxygen requirements before intubation, 1259 (89.7\%) were on traditional ventilators, $142(10.1 \%)$ were on converted BiPAP machines, and $2(0.1 \%)$ were on anesthesia machines. The length of time on mechanical ventilation was 0 to 7 days for $851 / 1403$ patients $(60.7 \%)$ and 8 days or more for $552 / 1403$ patients $(39.3 \%)$.

Prone positioning was documented for $756 / 2634$ patients (28.7\%), and 270/2634 patients (10.3\%) patients were terminally weaned. Do not resuscitate (DNR) orders were completed for $1631 / 2634$ patients $(61.9 \%)$. A palliative care consult was provided to $1014 / 2634$ patients $(38.5 \%)$. At the time of death, the level of care was ICU for $1299 / 2634$ patients $(49.3 \%)$ and non-ICU for $1335 / 2634$ patients $(50.7 \%)$.

\section{Patient Outcomes Based on RRT/CA Calls}

Of the 2634 patients, $1112(42.2 \%)$ had an RRT/CA call at a non-ICU level of care, while $1522(57.8 \%)$ did not. As shown in Tables 4-6, the RRT/CA group was significantly different from the non-RRT/CA group in terms of age, race, and 
comorbidities. Among patients between 60 and 79 years of age, $618 / 1112(55.6 \%)$ were in the RRT/CA group and 623/1522 $(40.9 \%)$ were in the non-RRT/CA group. In terms of race, there were significantly fewer White patients in the RRT/CA group (404/1112, 36.3\%, versus 852/1522, 56.0\%; $P<.001)$. The RRT/CA cohort had a significantly higher rate of patients with diabetes $(491 / 1112,44.2 \%$, versus 552/1522, 36.3\%; $P<.001)$. Patients in the RRT/CA cohort were more likely to be admitted from home $(926 / 1112,83.3 \%)$ than patients in the non-RRT/CA cohort $(969 / 1522,63.7 \%)$. Patients in the RRT/CA cohort were more likely than patients in the non-RRT/CA cohort to be admitted to a medical/surgical unit $(576 / 1112,51.8 \%$, versus $654 / 1522,42.9 \%)$ or telemetry/step-down unit $(455 / 1112$
$40.9 \%$, versus $408 / 1522,26.8 \%$ ), and to die at an ICU level of care $(671 / 1112,60.3 \%$, versus $628 / 1522,41.3 \%)$. An overall length of stay (LOS) of 8 days or more was more common in the RRT/CA cohort $(645 / 1112,58.0 \%)$ than in the non-RRT/CA cohort $(569 / 1522,37.4 \%)$, as was an ICU LOS of 0 to 7 days $(472 / 1112,42.0 \%$, versus $400 / 1522,26.3 \%)$ and of 8 days or more $(271 / 1112,24.4 \%$, versus $303 / 1522,19.9 \%)$. After adjusting for demographic and clinical characteristics, oxygen saturation levels at presentation were significant for the RRT/CA cohort at oxygen saturation levels of $80 \%$ to $89 \%$ (odds ratio [OR] 1.988, 95\% CI 1.511-2.616) and of $\geq 90 \%$ (OR 2.517, 95\% CI 1.962-3.230). For the logistic regression results, see Table 7 . 
Table 4. Baseline characteristics of patients who died of COVID-19 who experienced an RRT/CA call at a non-ICU level of care (N=2634).

\begin{tabular}{|c|c|c|c|}
\hline \multirow[t]{2}{*}{ Baseline characteristics } & \multicolumn{3}{|l|}{$\mathrm{RRT} / \mathrm{CA}^{\mathrm{a}}$ call } \\
\hline & Yes $(\mathrm{n}=1112), \mathrm{n}(\%)$ & No $(n=1522), n(\%)$ & $P$ value \\
\hline Age (years) & & & $<.001$ \\
\hline $21-39$ & $19(1.7)$ & $30(2.0)$ & \\
\hline $40-59$ & $194(17.5)$ & $157(10.3)$ & \\
\hline $60-79$ & $618(55.6)$ & $623(40.9)$ & \\
\hline$\geq 80$ & $281(25.3)$ & $712(40.8)$ & \\
\hline Sex & & & .35 \\
\hline Male & $714(64.2)$ & $950(62.4)$ & \\
\hline Female & $398(35.8)$ & $572(37.6)$ & \\
\hline Race & & & $<.001$ \\
\hline White & $404(36.3)$ & $852(56.0)$ & \\
\hline Black & $235(21.1)$ & $228(15.0)$ & \\
\hline Asian & $125(11.2)$ & $105(6.9)$ & \\
\hline Other/unknown & $348(31.3)$ & $337(22.1)$ & \\
\hline Payment method & & & $<.001$ \\
\hline Commercial insurance & $226(20.3)$ & $187(12.3)$ & \\
\hline Medicaid & $166(14.9)$ & $175(11.5)$ & \\
\hline Medicare & $702(63.1)$ & $1137(74.7)$ & \\
\hline Self-pay & $18(1.6)$ & $23(1.5)$ & \\
\hline \multicolumn{4}{|l|}{ Comorbidities } \\
\hline Hypertension & & & .24 \\
\hline Yes & $740(66.5)$ & $979(64.3)$ & \\
\hline No & $372(33.5)$ & $543(35.7)$ & \\
\hline COPD $^{b}$ & & & .08 \\
\hline Yes & $147(13.2)$ & $238(15.6)$ & \\
\hline No & $965(86.8)$ & $1284(84.4)$ & \\
\hline Diabetes & & & $<.001$ \\
\hline Yes & $491(44.2)$ & $552(36.3)$ & \\
\hline No & $621(55.9)$ & $970(63.7)$ & \\
\hline Heart failure & & & .03 \\
\hline Yes & $106(9.5)$ & $185(12.2)$ & \\
\hline No & $1006(90.5)$ & $1337(87.8)$ & \\
\hline Dementia & & & $<.001$ \\
\hline Yes & $98(8.8)$ & $333(21.9)$ & \\
\hline No & 1014 (91.2) & $1189(78.1)$ & \\
\hline End stage renal disease & & & .02 \\
\hline Yes & $85(7.6)$ & $81(5.3)$ & \\
\hline No & $1027(92.4)$ & $1441(94.7)$ & \\
\hline Number of comorbidities & & & .47 \\
\hline 0 & $202(18.2)$ & $243(15.9)$ & \\
\hline 1 & 355 (31.9) & $484(31.8)$ & \\
\hline
\end{tabular}




\begin{tabular}{|c|c|c|c|}
\hline \multirow[t]{2}{*}{ Baseline characteristics } & \multicolumn{3}{|l|}{$\mathrm{RRT} / \mathrm{CA}^{\mathrm{a}}$ call } \\
\hline & Yes $(\mathrm{n}=1112), \mathrm{n}(\%)$ & No $(\mathrm{n}=1522), \mathrm{n}(\%)$ & $P$ value \\
\hline 2 & 388 (34.9) & $546(35.9)$ & \\
\hline 3 & $134(12.1)$ & 209 (13.7) & \\
\hline 4 & $31(2.8)$ & $35(2.3)$ & \\
\hline 5 & $2(0.2)$ & $5(0.3)$ & \\
\hline BMI $\left(\mathbf{k g} / \mathbf{m}^{2}\right)$ & & & $<.001$ \\
\hline Unknown & $136(12.2)$ & $358(23.5)$ & \\
\hline$<18.5$ & $22(1.9)$ & $60(3.9)$ & \\
\hline $18.5-24.99$ & $236(21.2)$ & $352(23.1)$ & \\
\hline $25-29.99$ & $352(31.7)$ & $380(24.9)$ & \\
\hline $30-34.99$ & $206(18.5)$ & $195(12.8)$ & \\
\hline $35-39.99$ & 88 (7.9) & $102(6.7)$ & \\
\hline$\geq 40$ & $72(6.5)$ & $75(4.9)$ & \\
\hline
\end{tabular}

${ }^{a}$ RRT/CA: rapid response team/cardiac arrest.

${ }^{\mathrm{b}} \mathrm{COPD}$ : chronic obstructive pulmonary disease. 
Table 5. Hospitalization characteristics of patients who died of COVID-19 who experienced an RRT/CA call at a non-ICU level of care (N=2634).

\begin{tabular}{|c|c|c|c|}
\hline \multirow[t]{2}{*}{ Baseline characteristics } & \multicolumn{3}{|l|}{$\mathrm{RRT} / \mathrm{CA}^{\mathrm{a}}$ call } \\
\hline & Yes $(n=1112), n(\%)$ & No $(n=1522), n(\%)$ & $P$ value \\
\hline Admission source & & & $<.001$ \\
\hline Home & $926(83.3)$ & $969(63.7)$ & \\
\hline Rehabilitation & $34(3.0)$ & $93(6.1)$ & \\
\hline Skilled nursing facility & $80(7.2)$ & $331(21.7)$ & \\
\hline Transfer from another acute care hospital & $72(6.5)$ & $129(8.5)$ & \\
\hline
\end{tabular}

Emergency department visit

Within 48 hours of this admission

Yes

No

Within 7 days of this admission

$$
\text { Yes }
$$

No

Readmission

Within 24 hours

Yes

No

Within 7 days

Yes

No

Within 30 days

Yes

No

Level of care at time of death

$\mathrm{ICU}^{\mathrm{c}}$
Non-ICU

Level of care at time of admission

$\mathrm{ICU}$

Medical/surgical unit

Telemetry/stepdown unit

Overall length of stay (days)

0-7

$\geq 8$

ICU length of stay (days)

0-7

$\geq 8$

Oxygen saturation on presentation (\%)

$<80$

80-89.9

$\geq 90$

Unable to determine
$29(2.6)$

22 (1.5)

1083 (97.4)

1500 (98.6)

$61(5.5)$

$1051(94.5)$

7 (0.6)

1478 (97.1)

$1081(97.2)$

71 (6.4)

1041 (93.6)

$671(60.3)$

441 (39.7)

$81(7.3)$

$576(51.8)$

455 (40.9)

467 (42.0)

$645(58.0)$

472 (42.4)

$271(24.4)$

152 (13.7)

289 (26.0)

664 (59.7)

7 (0.6)
64 (4.2)

1458 (95.8)

.51

13 (0.9)

1509 (99.2)

44 (2.9)

123 (8.1)

.13

.88

.10

1399 (91.9)

$N / A^{b}$

$628(41.3)$

894 (58.7)

$<.001$

460 (30.2)

654 (42.9)

408 (26.8)

$<.001$

953 (62.6)

569 (37.4)

$<.001$

400 (26.3)

303 (19.9)

$<.001$
307 (20.2)

378 (24.8)

814 (53.5)

23 (1.5) 


\begin{tabular}{|c|c|c|c|}
\hline \multirow[t]{2}{*}{ Baseline characteristics } & \multicolumn{3}{|l|}{$\mathrm{RRT} / \mathrm{CA}^{\mathrm{a}}$ call } \\
\hline & Yes $(\mathrm{n}=1112), \mathrm{n}(\%)$ & No $(\mathrm{n}=1522), \mathrm{n}(\%)$ & $P$ value \\
\hline Initial respiratory support on presentation & & & $<.001$ \\
\hline None & $687(61.8)$ & $710(46.7)$ & \\
\hline Nasal cannula & $161(14.5)$ & $202(13.3)$ & \\
\hline High-flow nasal cannula & $0(0.0)$ & $8(0.5)$ & \\
\hline Ventimask & $2(0.2)$ & $9(0.6)$ & \\
\hline BiPAP $^{d}$ & $2(0.2)$ & $11(0.7)$ & \\
\hline Nonrebreather mask & $239(21.5)$ & $503(33.1)$ & \\
\hline Ventilator & $1(0.1)$ & $23(1.5)$ & \\
\hline Other & $4(0.4)$ & $23(1.5)$ & \\
\hline Unable to determine & $16(1.4)$ & $33(2.2)$ & \\
\hline Mechanical ventilation & $723(65.0)$ & $680(44.7)$ & $<.001$ \\
\hline \multicolumn{4}{|l|}{ Type of mechanical ventilation } \\
\hline Traditional ventilator & $650(58.5)$ & $609(40.0)$ & \\
\hline Converted BiPAP & $71(6.4)$ & $71(4.7)$ & \\
\hline Anesthesia machine & $2(0.2)$ & $0(0.0)$ & \\
\hline Increased oxygen requirement before mechanical ventilation & $699(62.9)$ & $633(41.6)$ & $<.001$ \\
\hline \multicolumn{4}{|l|}{ Mechanical ventilation length (days) } \\
\hline $0-7$ & $461(41.5)$ & $390(25.6)$ & \\
\hline$\geq 8$ & $262(23.6)$ & $290(19.1)$ & \\
\hline Terminal wean & & & .52 \\
\hline Yes & $109(9.8)$ & $161(10.6)$ & \\
\hline No & $1003(90.2)$ & $1361(89.4)$ & \\
\hline Proning & & & $<.001$ \\
\hline Yes & $500(45.0)$ & $256(16.8)$ & \\
\hline No & $612(54.9)$ & $1266(83.2)$ & \\
\hline Proning without mechanical ventilation & $116(10.4)$ & $75(4.9)$ & \\
\hline Proning before mechanical ventilation & $171(15.4)$ & $42(2.7)$ & \\
\hline Proning during mechanical ventilation & $99(8.9)$ & $115(7.5)$ & \\
\hline Proning before and during mechanical ventilation & $114(10.3)$ & $24(1.6)$ & \\
\hline DNR $^{\mathrm{e}}$ complete & & & $<.001$ \\
\hline Yes & $558(50.2)$ & $1073(70.5)$ & \\
\hline No & $554(49.8)$ & 449 (29.5) & \\
\hline Palliative care consult & & & $<.001$ \\
\hline Yes & 385 (34.6) & $629(41.3)$ & \\
\hline No & $727(65.4)$ & $893(58.7)$ & \\
\hline Clinical trial inclusion & & & N/A \\
\hline Yes & $91(8.2)$ & $23(1.5)$ & \\
\hline
\end{tabular}




\begin{tabular}{lll}
\hline Baseline characteristics & RRT/CA ${ }^{\mathrm{a}}$ call & \\
& Yes $(\mathrm{n}=1112), \mathrm{n}(\%)$ & No (n=1522), n (\%) \\
\hline No & $1021(91.8)$ & $P$ value \\
\hline
\end{tabular}

${ }^{\mathrm{a}} \mathrm{RRT} / \mathrm{CA}$ : rapid response team/cardiac arrest.

${ }^{b}$ N/A: not applicable.

${ }^{\mathrm{c}} \mathrm{ICU}$ : intensive care unit.

${ }^{\mathrm{d}}$ BiPAP: bilevel positive airway pressure.

${ }^{\mathrm{e}}$ DNR: do not resuscitate.

Table 6. Additional characteristics associated with RRT/CA calls for patients at a non-intensive care unit level of care ( $\mathrm{n}=1112)$, n (\%).

\begin{tabular}{|c|c|}
\hline Characteristic & Value \\
\hline Required escalation in level of care following initial $\mathrm{RRT} / \mathrm{CA}^{\mathrm{a}}$ call & $716(64.4)$ \\
\hline \multicolumn{2}{|l|}{ Oxygen saturation at time RRT/CA call initiated (\%) } \\
\hline$<80$ & $479(43.1)$ \\
\hline $80-89$ & $407(36.6)$ \\
\hline$\geq 90$ & $128(11.5)$ \\
\hline Unable to determine & $98(8.8)$ \\
\hline \multicolumn{2}{|l|}{ Oxygen supplement at time RRT/CA call initiated } \\
\hline Nonrebreather mask with or without nasal cannula & $868(78.1)$ \\
\hline Nasal cannula & $147(13.2)$ \\
\hline Room air & $40(3.6)$ \\
\hline Ventimask & $18(1.6)$ \\
\hline Ventilator & $11(1.0)$ \\
\hline High-flow nasal cannula & $9(0.8)$ \\
\hline $\mathrm{BiPAP}^{\mathrm{b}}$ & $5(0.4)$ \\
\hline Unable to determine & $14(1.3)$ \\
\hline \multicolumn{2}{|l|}{ Most recent oxygen saturation before RRT/CA initiated (\%) } \\
\hline$<80$ & $43(3.9)$ \\
\hline $80-89$ & $211(18.9)$ \\
\hline $90 \leq$ & $852(76.6)$ \\
\hline Unable to determine & $6(0.5)$ \\
\hline \multicolumn{2}{|c|}{ Documented timing of most recent oxygen saturation before RRT/CA initiated (hours) } \\
\hline$<1$ & $263(23.7)$ \\
\hline $1-2$ & $191(17.2)$ \\
\hline $2-3$ & $140(12.6)$ \\
\hline $3-4$ & $109(9.8)$ \\
\hline$>4$ & $409(36.8)$ \\
\hline
\end{tabular}

${ }^{a}$ RRT/CA: rapid response team/cardiac arrest.

${ }^{b}$ BiPAP: bilevel positive airway pressure. 
Table 7. Regression analysis of patients who died of COVID-19 who experienced a rapid response team/cardiac arrest call at a non-intensive care unit level of care $(\mathrm{N}=2634)$.

\begin{tabular}{|c|c|c|c|c|}
\hline Baseline characteristics & Estimate & $P$ value & Odds ratio & $95 \% \mathrm{CI}$ \\
\hline \multicolumn{5}{|l|}{ Age (years) } \\
\hline $50-69$ & 0.2653 & .20 & 1.304 & $0.872-1.949$ \\
\hline $70-79$ & 0.1721 & .44 & 1.188 & $0.766-1.842$ \\
\hline$\geq 80$ & -0.3179 & .17 & 0.728 & $0.460-1.151$ \\
\hline \multicolumn{5}{|l|}{ Sex } \\
\hline Male & -0.2299 & .02 & 0.795 & $0.658-0.960$ \\
\hline \multicolumn{5}{|l|}{ Race } \\
\hline Black & 0.6134 & $<.001$ & 1.847 & $1.445-2.361$ \\
\hline Asian & 0.6548 & $<.001$ & 1.925 & $1.395-2.655$ \\
\hline Other/unknown & 0.5333 & $<.001$ & 1.704 & $1.362-2.133$ \\
\hline \multicolumn{5}{|l|}{ Payment method } \\
\hline Medicaid & -0.0458 & .78 & 0.955 & $0.691-1.321$ \\
\hline Medicare & -0.0107 & .94 & 0.989 & $0.750-1.305$ \\
\hline Self-pay & -0.3020 & .40 & 0.739 & $0.367-1.488$ \\
\hline \multicolumn{5}{|l|}{ Comorbidities } \\
\hline Heart failure & 0.1429 & .34 & 1.154 & $0.860-1.547$ \\
\hline End stage renal disease & 0.6184 & .002 & 1.856 & $1.262-2.729$ \\
\hline $\mathrm{COPD}^{\mathrm{a}}$ & -0.1216 & .35 & 0.886 & $0.687-1.141$ \\
\hline Hypertension & 0.1239 & .21 & 1.132 & $0.931-1.376$ \\
\hline Diabetes mellitus & 0.0833 & .38 & 1.087 & $0.902-1.310$ \\
\hline \multicolumn{5}{|l|}{ BMI $\left(\mathrm{kg} / \mathrm{m}^{2}\right)$} \\
\hline Unknown & -0.4645 & $<.001$ & 0.628 & $0.491-0.804$ \\
\hline$\geq 30$ & -0.0545 & .62 & 0.947 & $0.765-1.173$ \\
\hline \multicolumn{5}{|l|}{ Admit source } \\
\hline Home & 0.9060 & $<.001$ & 2.474 & $1.850-3.310$ \\
\hline Rehabilitation & 0.2904 & .25 & 1.337 & $0.813-2.199$ \\
\hline Transfer from acute care hospital & 0.0544 & .80 & 1.056 & $0.691-1.614$ \\
\hline \multicolumn{5}{|l|}{ Oxygen saturation on presentation (\%) } \\
\hline $80-89$ & 0.6871 & $<.001$ & 1.988 & 1.5112 .616 \\
\hline$\geq 90$ & 0.9232 & $<.001$ & 2.517 & 1.9623 .230 \\
\hline Proning & 1.1840 & $<.001$ & 3.267 & 2.6674 .003 \\
\hline
\end{tabular}

${ }^{\mathrm{a}} \mathrm{COPD}$ : chronic obstructive pulmonary disease.

\section{Discussion}

\section{Summary of Findings}

This study represents a review of one of the largest cohorts of COVID-19 mortality that includes data documented in nonstructured fields within the EHR. An experienced team of registered nurses was able to extract detailed information from the medical record that is typically not included in a structured data set analysis. The demographics of the patients who died are similar to those in other published studies: age predominately over 69, male majority, payor mix (reflecting age and Medicare along with a low number of self-paying patients, namely 41/2634, 1.6\%), and multiple comorbidities [3-12].

\section{Circumstances Preceding Patient Deterioration}

This study provides a detailed clinical picture of the circumstances that precede the sudden deterioration in non-ICU patients reported by clinicians, which have not been fully examined in the literature. A striking reported feature of COVID-19 is the rapid progression of respiratory failure soon after the onset of dyspnea and hypoxemia [13]. The US National Institutes of Health (NIH) has reported that hypoxemia is 
common in hospitalized patients with COVID-19 and that the criteria for hospital admission, ICU admission, and mechanical ventilation differ between countries [14]. In some hospitals in the United States, more than $25 \%$ of hospitalized patients require ICU care, mostly due to acute respiratory failure. The NIH recommends close monitoring for worsening respiratory status for adults with COVID-19 who are receiving supplemental oxygen. These recommendations align with our findings in the non-ICU patient population.

Approximately half of the deaths (1335/2634, 50.7\%) occurred at a non-ICU level of care despite admission to the appropriate care setting with normal staffing. Our analysis of patients who experienced at least one RRT/CA call at a non-ICU level of care revealed that $716 / 1112(64.4 \%)$ required an escalation in their level of care. Of the RRT/CA patients, 664/1112 (59.7\%) presented to the hospital with oxygen saturation levels greater than or equal to $90 \%$. In addition, 687/1112 (61.8\%) had no oxygen support. Of the RTT/CA patients, 1031/1112 (92.7\%) were admitted to a non-ICU level of care with normal staffing levels, which was appropriate based on their care needs. At presentation to the ED, the oxygen saturation levels for these patients were significantly higher than those for patients admitted to the ICU. Before the RRT/CA call, the most recent oxygen saturation levels recorded for the non-ICU patients remained high, at $\geq 90 \%$ for $852 / 1112(76.6 \%)$ of patients. Oxygen saturations were documented within two hours of the RRT/CA call in 454/1112 (40.9\%) of patients in the RRT/CA cohort. When the RRT/CA was called, 479/1112 (43.1\%) of patients had an oxygen saturation less than $80 \%$, and $78.1 \%$ $(868 / 1112)$ were on a nonrebreather mask or a nonrebreather mask with nasal cannula. These data imply a sudden, unexpected deterioration in respiratory status requiring an RRT/CA call in a large number of non-ICU patients.

\section{Limitations}

This study includes the following limitations. First, the study focuses on the demographic and clinical characteristics of in-hospital COVID-19 patients who died between March 13 and April 30, 2020; it does not provide a comparison group of similar patients who survived during the same time period. Second, data were obtained from the EHR and manually abstracted from medical records through retrospective review; however, some routine documentation was less detailed due to the volume of patients being treated. Third, race was documented as other/unknown in 685/2634 (26\%) of patients; therefore, conclusions about race could not be drawn. Fourth, missing BMI data were included in the category of "unknown" BMI. Finally, the study does not recognize a specific trigger that can distinguish which non-ICU patients in the cohort should be monitored.

\section{Conclusions}

Patients admitted to a non-ICU level of care appear to suffer rapid clinical deterioration, often with the hallmark of a sudden decrease in oxygen saturation. This finding suggests that non-ICU patients could benefit from additional monitoring, such as continuous central oxygenation saturation. The availability of wireless patch monitoring should be considered along with other methods, such as carbon dioxide and cardiac monitoring. Although this approach does not ensure reduced mortality, the number of RRT/CA calls infers that this area warrants further study.

\section{Acknowledgments}

This work was supported by grants R24AG064191 from the National Institute on Aging of the National Institutes of Health and R01LM012836 from the National Library of Medicine of the National Institutes of Health. We thank the Northwell Health Institute for Clinical Excellence and Quality/Safety: Mary Cama, RN, MSN; Patricia Meo, RN, BSN; Amy Logeman, RN, BSN, CPPS; Maureen McCarthy, RN, BSN; Josephine Fernandez-Kapilevich, RN, BSN; Theresa A Droluk, RN, BSN; Jessica Martin, RN, BSN, RN-BC; Allison Carballo, RN, MBA; and Jimmy Diaz, BS. We also thank the Northwell Health Krasnoff Quality Management Institute: Alex Ma; Kahliik S Burrell; and Mark P Tursi, MBA. We would like to acknowledge the contributions of the Northwell Health COVID-19 Research Consortium, including Crystal R Herron, PhD, and Jennifer C Johnson, MS, for editorial support. We acknowledge and honor all our Northwell Health team members who consistently put themselves in harm's way during the COVID-19 pandemic. We dedicate this article to them, as their vital contribution to knowledge about COVID-19 and sacrifices on the behalf of patients made it possible.

\section{Authors' Contributions}

MPJ had full access to all data in the study and takes responsibility for the integrity of the data and the accuracy of the data analysis. MPJ, SES, JSL, and KLN were responsible for the conception and design of the study. MPJ, SES, JSL, JJW, LS, MDG, and KLN were responsible for data acquisition, analysis, and interpretation. MPJ, SES, JSL, JJW, LS, and KLN were responsible for drafting the manuscript. MPJ, SES, JSL, JJW, LS, MDG, and KLN were responsible for critical revision of the manuscript for important intellectual content. JJW was responsible for the statistical analysis. MPJ, SES, JSL, JJW, LS, MDG, and KLN were responsible for administrative, technical, and material support. MPJ supervised the study.

\section{Conflicts of Interest}

None declared.

\section{References}


1. COVID-19 Dashboard. The Center for Systems Science and Engineering at Johns Hopkins University. 2020. URL: https:/ /gisanddata.maps.arcgis.com/apps/opsdashboard/ [accessed 2020-03-24]

2. COVID-19 Tracker. New York State Department of Health. 2020. URL: https://covid19tracker.health.ny.gov/views/ NYS-COVID19-Tracker/NYSDOHCOVID-19Tracker-Map?\%3Aembed=yes\&\%3Atoolbar=no\&\%3Atabs=n\#/views [accessed 2020-05-07]

3. CDC COVID-19 Response Team. Preliminary Estimates of the Prevalence of Selected Underlying Health Conditions Among Patients with Coronavirus Disease 2019 - United States, February 12-March 28, 2020. MMWR Morb Mortal Wkly Rep 2020 Apr 03;69(13):382-386 [FREE Full text] [doi: 10.15585/mmwr.mm6913e2] [Medline: 32240123]

4. Wortham JM, Lee JT, Althomsons S, Latash J, Davidson A, Guerra K, et al. Characteristics of Persons Who Died with COVID-19 - United States, February 12-May 18, 2020. MMWR Morb Mortal Wkly Rep 2020 Jul 17;69(28):923-929 [FREE Full text] [doi: 10.15585/mmwr.mm6928e1] [Medline: 32673298]

5. Wang C, Horby P, Hayden F, Gao G. A novel coronavirus outbreak of global health concern. Lancet 2020 Feb;395(10223):470-473. [doi: 10.1016/S0140-6736(20)30185-9]

6. Richardson S, Hirsch JS, Narasimhan M, Crawford JM, McGinn T, Davidson KW, the Northwell COVID-19 Research Consortium, et al. Presenting Characteristics, Comorbidities, and Outcomes Among 5700 Patients Hospitalized With COVID-19 in the New York City Area. JAMA 2020 May 26;323(20):2052-2059 [FREE Full text] [doi: 10.1001/jama.2020.6775] [Medline: 32320003]

7. Zhou F, Yu T, Du R, Fan G, Liu Y, Liu Z, et al. Clinical course and risk factors for mortality of adult inpatients with COVID-19 in Wuhan, China: a retrospective cohort study. Lancet 2020 Mar;395(10229):1054-1062 [FREE Full text] [doi: 10.1016/S0140-6736(20)30566-3]

8. Goyal P, Choi J, Pinheiro L, Schenck EJ, Chen R, Jabri A, et al. Clinical Characteristics of Covid-19 in New York City. N Engl J Med 2020 Jun 11;382(24):2372-2374 [FREE Full text] [doi: 10.1056/NEJMc2010419] [Medline: 32302078]

9. Petrilli C, Jones S, Yang J, Rajagopalan H, O'Donnell L, Chernyak Y, et al. Factors associated with hospital admission and critical illness among 5279 people with coronavirus disease 2019 in New York City: prospective cohort study. BMJ 2020 May 22;369:m1966. [doi: 10.1136/bmj.m1966] [Medline: 32444366]

10. Garg S, Kim L, Whitaker M, O'Halloran A, Cummings C, Holstein R, et al. Hospitalization Rates and Characteristics of Patients Hospitalized with Laboratory-Confirmed Coronavirus Disease 2019 - COVID-NET, 14 States, March 1-30, 2020. MMWR Morb Mortal Wkly Rep 2020 Apr 17;69(15):458-464 [FREE Full text] [doi: 10.15585/mmwr.mm6915e3] [Medline: $\underline{32298251]}$

11. Stokes EK, Zambrano LD, Anderson KN, Marder EP, Raz KM, El Burai Felix S, et al. Coronavirus Disease 2019 Case Surveillance - United States, January 22-May 30, 2020. MMWR Morb Mortal Wkly Rep 2020 Jun 19;69(24):759-765. [doi: 10.15585/mmwr.mm6924e2] [Medline: 32555134]

12. Yancy CW. COVID-19 and African Americans. JAMA 2020 May 19;323(19):1891-1892. [doi: 10.1001/jama.2020.6548] [Medline: 32293639]

13. Berlin DA, Gulick RM, Martinez FJ. Severe Covid-19. N Engl J Med 2020 May 15:online. [doi: 10.1056/nejmcp2009575]

14. COVID-19 Treatment Guidelines. US National Institutes of Health. URL: https://www.covid19treatmentguidelines.nih.gov/ introduction/ [accessed 2020-04-24]

\author{
Abbreviations \\ DNR: do not resuscitate \\ ED: emergency department \\ EHR: electronic health record \\ ICU: intensive care unit \\ LOS: length of stay \\ NIH: National Institutes of Health \\ OR: odds ratio \\ RRT/CA: rapid response team/cardiac arrest
}

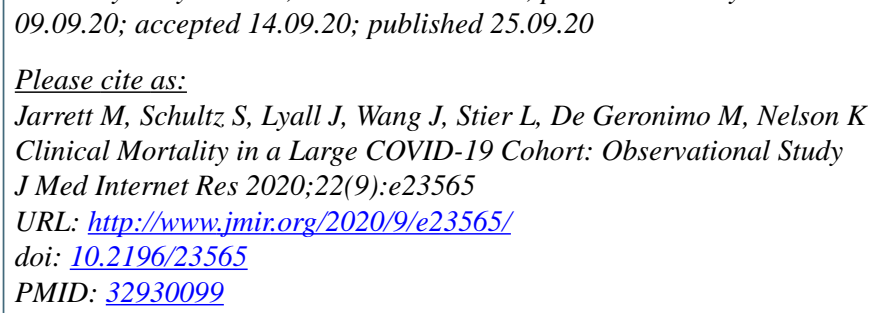


CMark Jarrett, Susanne Schultz, Julie Lyall, Jason Wang, Lori Stier, Marcella De Geronimo, Karen Nelson. Originally published in the Journal of Medical Internet Research (http://www.jmir.org), 25.09.2020. This is an open-access article distributed under the terms of the Creative Commons Attribution License (https://creativecommons.org/licenses/by/4.0/), which permits unrestricted use, distribution, and reproduction in any medium, provided the original work, first published in the Journal of Medical Internet Research, is properly cited. The complete bibliographic information, a link to the original publication on http://www.jmir.org/, as well as this copyright and license information must be included. 